\title{
CO-PRESENCIAS Y VINCULACIONES EN RED: LAS RELACIONES INTERDEPENDIENTES EN LA ENSEÑANZA Y EL APRENDIZAJE DEL DIBUJO EN EL NIVEL SUPERIOR UNIVERSITARIO
}

\author{
Por Susana H. Mattanó* \\ Universidad Nacional de Rosario, Argentina. \\ smattano@unr.edu.ar
}

Recibido: 29/06/2014 Aceptado: 03/11/2014

\section{Resumen}

El arte y la educación artística -al igual que las personas- se caracterizan por relaciones de co-presencia opuestas, complementarias e interdependientes. La enseñanza y el aprendizaje del Dibujo en el Nivel Superior Universitario necesita ser abordada teniendo en cuenta que esas relaciones interdependientes atraviesan no sólo contenidos específicos del lenguaje visual sino también a los textos verbales y no verbales; a los sujetos lectores y productores; a docentes, alumnas y alumnos, quienes a su vez, construyen saberes y forman parte de sociedades, estructurados y dinamizados a través de conexiones en red. La metodología de enseñanza y de aprendizaje del Dibujo, no debe dejar de considerar la pérdida de límites y fronteras de las disciplinas artísticas como reflejo de la cultura contemporánea y de las relaciones interdependientes entre sujetos/s, texto/s y contexto/s. Debe generar la construcción de un espacio de acción en donde alumnas y alumnos expresen y comuniquen ideas y vivencias en sus dibujos, pero además, den y generen opinión sobre las problemáticas que aborda el arte contemporáneo a través de proyectos artísticos que tiendan a la cooperación social, a la solución de problemas y al bien común.

\section{Palabras clave:}

Enseñanza - Aprendizaje - Dibujo - Red - Relaciones interdependientes.

\footnotetext{
Especialista en Política y Gestión de la Educación Superior (Centro de Estudios Interdisciplinarios - UNR.), Licenciada en Bellas Artes (Facultad de Humanidades y Artes - UNR), Profesora Nacional en Artes Visuales (Facultad de Humanidades y Artes - UNR). Docente Titular de Dibujo (Escuela de Bellas Artes UNR).
} 
Revista de la Escuela de Ciencias de la Educación, año 11, número 10, enero a diciembre de 2015. PÁginas 43-62. ISSN 1851-6297. ISSN EN LÍNEA 2362-3349. CO-PRESENCIAS Y VINCULACIONES EN RED: LAS RELACIONES INTERDEPENDIENTES EN LA ENSEÑANZA Y EL APRENDIZAJE DEL DIBUJO EN EL NIVEL SUPERIOR UNIVERSITARIO. SUSANA H. MATTANó

\begin{abstract}
Art and Artistic Education -the same as persons- are characterized by oposite, complementary and inter-dependent relationships of co-presence. Teaching and learning Drawing at the University needs to be board knowing that inter-dependent relationships go through not only by specific contents of visual language but also they are in verbal and unverbal texts; between producers and readers; between teachers and students which built knoledges and belong to societies structured and dynamized by net connections. The methodolgy of teaching and learning Drawing contents, must not leave to considerate how artistics disciplines are loosing limits and frontiers as reflex of contemporary culture and inter-dependent relationships between subject/s, text/s and context/s. It must generate the construction of an space of action for students could express and communicate ideas and life experiencies on their drawings, and also where they could give and generate opinions about problematics of contemporary art through artistic proyects wich tend to social colaboration, problems solutions and popular good.
\end{abstract}

\title{
Key words:
}

Teaching - Learning - Drawing - Net - Inter-dependent Relationships.

"Supongamos que, a pesar de la incesante variedad de prácticas que pueblan el variabilísimo

presente, pueden proponerse generalizaciones que nos permitan agudizar nuestras observaciones y refinar nuestras ideas sobre lo que constituye la singularidad de las artes de estos años." Laddaga, R. (2010, p. 5)

Desde hace tiempo, el debate en torno a la desaparición de las disciplinas artísticas fluctúa entre la caducidad del Dibujo, la Pintura, la Escultura, el Grabado -como categorías de simbolización artística- y la aparición de otras expresiones, que al no pertenecer a ninguna de las categorías citadas, son denominadas como acciones, eventos, proyectos participativos, intervenciones, etc.

Los artistas que se consagran a estas prácticas no abandonan simplemente los objetivos anteriores de la práctica artística -construir objetos o diseñar procesos que sean semánticamente densos y formalmente complejos-, sino que intentan vincular estos objetivos a la edificación de aparatos socio-técnicos que permitan que se desplieguen procesos de aprendizaje colectivo, en los cuales la tradición del arte moderno sea confrontada en forma directa con las problemáticas de sitios sociales particulares (Ladagga, 2010, pp.129-130).

Una mirada sobre esta situación nos confronta con miles de autores y producciones artísticas que instalan al arte en la esfera de "lo moderno" frente 
Revista de la Escuela de Ciencias de la Educación, año 11, número 10, enero a diciembre de 2015. Páginas 43-62. ISSN 1851-6297. ISSN EN LINEA 2362-3349. Co-PRESENCIAS Y VINCULACIONES EN RED: LAS RELACIONES INTERDEPENDIENTES

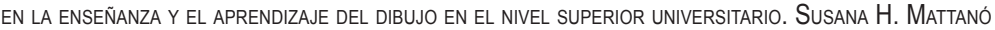

a otros tantos que abandonan esos modos de representación. Más que preguntarnos de qué se trata y respondernos que es una época de transición (cosa imposible de corroborar); más que adoptar una u otra postura, podríamos intentar definir a la nuestra como una época de co-presencia, de convivencia -en diálogo sincrónico- entre categorías simbólicas establecidas y nuevas formas artísticas que señalan diferencias; que suman y pierden adeptos y críticos, construyendo saberes y nuevas estructuras culturales y sociales conectados en forma de red (1). Esta co-presencia de paradigmas (2) se refleja en una co-existencia cultural y social entre las producciones artísticas en galerías y museos, y las transiciones que construyen proyectos y experiencias contemporáneos "entre el espacio de las galerías o museos y el lugar donde tienen lugar esas operaciones entre expertos y no expertos que producen manipulaciones de imágenes o símbolos y modificaciones directas en las relaciones entre los cuerpos" (Ladagga, 2006, p.263). Esta sincronía de paradigmas está presente en la Escuela de Arte de la Facultad de Humanidades y Artes de la Universidad Nacional de Rosario, y se visualiza tanto en la formación y posturas de docentes como así también en los intereses y tendencias de producción artística entre los estudiantes. Es necesario remarcar que la pérdida de límites entre las disciplinas artísticas no es un hecho aislado y casual y responde a un proceso de concordancia que se da con cambios culturales, institucionales, económicos, tecnológicos, políticos a nivel mundial, partir de las últimas décadas del siglo veinte.

El proceso de desborde de ese universo y de lenta construcción de otro (por debilitamiento de la soberanía de los Estados nacionales, por la multiplicación de las formas de ciudadanía compleja, por la constitución de redes transnacionales de activismo o de protesta, por la conciencia de crisis ecológica, por la diversificación de las conexiones) es lo solemos llamar globalización. (Ladagga, 2006, p.8).

Y es, precisamente, la globalización "un conjunto de procesos de conexión y desconexión" (Ladagga, 2006, p. 49); no hay una desaparición de las formas sociales sino una pérdida de frontera de las Naciones. Estos procesos de conexión y desconexión pueden ser la mirada aplicable a las disciplinas artísticas: hay producciones que no pueden ser definidas específicamente en una de las categorías simbólicas del arte moderno (Dibujo, Pintura, Escultura, Grabado) y en las que se observa la pérdida de fronteras, el devenir en relación a otras; la condición de pertenecer a más de una -sin ser precisamente ninguna- pero que sin excluirse conviven en la escena actual. La reflexión de Laddaga sobre los límites del hombre de la modernidad en cuanto a la familia, la profesión, la identidad nacional -hoy disueltos en situaciones familiares complejas, inestabilidad laboral, nacionalidades múltiples- nos permite una clara imagen de la relación entre los cambios sociales, políticos y culturales que nos introducen en la comprensión de la pérdida de límites en el arte. 
Revista de la Escuela de Ciencias de la Educación, año 11, número 10, enero a diciembre de 2015. PÁginas 43-62. ISSN 1851-6297. ISSN EN LINEA 2362-3349. Co-PRESENCIAS Y VINCULACIONES EN RED: LAS RELACIONES INTERDEPENDIENTES

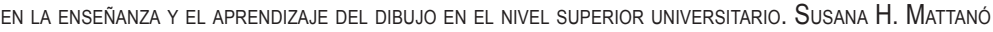

Es conveniente considerar también que, en esa pérdida de límites que atraviesa al sujeto de nuestro tiempo como persona y a su contexto, ninguno escapa al concepto de red que vincula y des-vincula al mismo tiempo.

El concepto de red, de amplia difusión en nuestra vida cotidiana (red neuronal; red social; red de establecimientos; red de intercambio; red inmobiliaria; red comunicacional; red energética; red de transporte; etc.) no es más que la verbalización de la imagen simbólica de una red como conjunto de lazos o uniones a través de nudos. "La red nos rodea, nos atraviesa, nos integra, nos atrapa y compromete. Nos permite conectar y crecer en experiencias y conocimientos a través de sus múltiples circuitos" (Ohlenschläger, 2009, p. 21). Una difundida publicidad de nuestro medio referida a internet, a la extorsión sexual y a la violencia, reza: "Internet es una red para estar unidos, comunicados, entretenidos. Conéctate con responsabilidad. La red nos une; que la red no te enrede"; en precisa alusión a la red de pesca que "atrapa" a algunos y no a otros, y a su forma de trama que une y separa simultáneamente. Sin duda alguna, un ejemplo claro y contemporáneo de vinculación/desvinculación como relación interdependiente. Podríamos también citar como ejemplo, a las redes comunicacionales establecidas por los avances tecnológicos: "las redes de computadoras se extienden tanto en la trama de la vida cotidiana que se vuelve incierto el límite entre el espacio de las imágenes y palabras digitalizadas y el de las cosas sólidas"(Ladagga, 2006, p.53). Los cambios tecnológicos han propiciado grandes avances en la vida cotidiana pero también un innegable cambio de percepción de las relaciones humanas en donde se dibuja una especie de destino común, compartido -a través de la virtualidad- que a su vez implica una pérdida de territorio. Estamos -a través de las imágenes y las comunicaciones virtuales- en otro sitio y en el nuestro al mismo tiempo, desdibujándose permanentemente los límites del propio espacio, ofreciendo un vasto material para el debate sobre la autoría (3).

La convergencia digital introduce en las políticas culturales una profunda renovación del modelo de comunicabilidad, pues del unidireccional, lineal y autoritario paradigma de la transmisión de información, hemos pasado al modelo de la red, esto es, al de la conectividad y la interacción que transforma la forma mecánica de la comunicación a distancia por la electrónica del interfaz de proximidad. Nuevo paradigma que se traduce en una política que privilegia la sinergia entre muchos pequeños proyectos, por sobre la complicada estructura de los grandes y pesados aparatos tanto en la tecnología como en la gestión (Bashiron Mendolicchio, 2014, p.1).

Prueba de ello son el surgimiento y la articulación de nuevos nodos y redes entre artistas e individuos en general, que aún sin conocerse personalmente comparten e intercambian redes temporales de distinta índole y diversidad. 
Revista de la Escuela de Ciencias de la Educación, año 11, número 10, enero a diciembre de 2015. Páginas 43-62. ISSN 1851-6297. ISSN EN LINEA 2362-3349. Co-PRESENCIAS Y VINCULACIONES EN RED: LAS RELACIONES INTERDEPENDIENTES

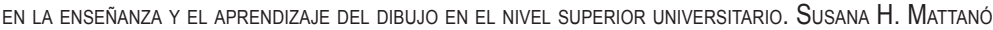

El modelo colaborativo de las Redes, más allá de su estética participativa e interconectada, se está afirmando también gracias a la actual crisis -o replanteamiento, reformulación- económica y a la consiguiente reestructuración sistémica de nuestra existencia. Compartir se convierte en una fórmula de ahorro, de redistribución, de exploración y explotación inteligente y sostenible de recursos, materiales, relaciones y conocimientos. El sistema red $-\mathrm{y}$ su evolución- está evidentemente vinculado a diversos aspectos tecnológicos, pero hablar de redes hoy no significa enfocarse exclusivamente hacia los elementos técnicos y comunicativos que las conforman o definen, sino más bien significa hablar de personas, de individuos y de colectivos, del desarrollo de las relaciones humanas y de los lazos económicos, políticos, sociales y culturales que comparten (Bashiron Mendolicchio, 2014, p.4).

Ante este estado de cuestión deberíamos preguntarnos más que por el rol de las escuelas de arte, por sus planes de estudios; si los contenidos y las metodologías de enseñanza y aprendizaje desarrolladas responden al nuevo escenario universal y al de nuestra vida cotidiana. $\mathrm{Y}$, en referencia al contenido específico de este escrito, los cuestionamientos serían: ¿cómo generar sentido en la enseñanza del Dibujo como disciplina artística en el Nivel Superior Universitario - de acuerdo a la categorización simbólica de los espacios curriculares de los planes de estudio actuales- cuando estamos en presencia de un nuevo paradigma artístico, social y cultural que implica la participación, la cooperación, la interrelación? ¿cuáles serían los modos de abordar el Enseñar/ el Aprender/ el Cursar/ el Hacer Dibujo en la Carrera de Bellas Artes (de la Facultad de Humanidades y Artes de la Universidad Nacional de Rosario) con el propósito de convertirse para todos -alumnas, alumnos y docentes-en experiencias creativas y estimulantes capaces de ofrecer diversos escenarios que se inserten y contemplen las problemáticas contemporáneas que nos atraviesan como actores en el mundo y en el campo de las artes? ¿Cómo ofrecer nuevas miradas sobre estrategias didácticas, contenidos y metodologías de enseñanza y aprendizaje del Dibujo que sean verdaderamente útiles para el futuro profesional como docentes y como artistas plásticos y que además, contemplen la pluralidad de disciplinas; la multiplicidad y la interrelación de lenguajes; los nuevos escenarios y medios de expresión y comunicación?

En primer término es necesario revalorizar el lugar de la enseñanza y aprendizaje del Dibujo dentro de la Carrera de Bellas Artes antes citada, muchas veces desplazado a un rol complementario a modo de sostén o punto de partida de otras disciplinas como la Pintura, el Grabado o la Escultura. Luego, recordar que en el ámbito universitario se desarrolla conocimiento y se reflexiona críticamente y que ésto puede hacerse también a través de la enseñanza y del aprendizaje del Dibujo. 
Revista de la Escuela de Ciencias de la Educación, año 11, número 10, enero a diciembre de 2015. PÁginas 43-62. ISSN 1851-6297. ISSN EN LINEA 2362-3349. CO-PRESENCIAS Y VINCULACIONES EN RED: LAS RELACIONES INTERDEPENDIENTES

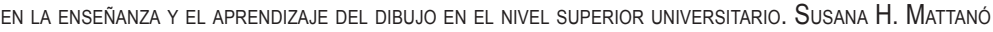

Darle tiempo al pensamiento y preocuparse por generar un pensamiento rico no son problemas de las ciencias sociales y humanas solamente o de algún nivel del sistema educativo, sino que son cuestiones que es posible extender tanto a las direrentes áreas como a todos los niveles. Las enseñanzas más elementales, los estudios básicos y los más complejos requieren ser aprendidos desde una perspectiva superior. Esto alude a que su solidez en tanto conocimiento implica que puedan ser explicativos de otros temas o campos, se pueda argumentar sobre ellos o permitan resolver problemas genuinos en un marco disciplinar (Litwin, 2012, p.102).

Además, es ineludible considerar al Dibujo como dimensión de ampliación y desarrollo de la capacidad perceptiva y creativa, de la comprensión racional y la manifestación sensible. Como producto de acciones que implican y necesitan tanto de la reflexión teórica como de la práctica en todas las instancias de ejecución. Como procedimiento que define ideas y medio instrumental para configurarlas y sobre todo como fin en sí mismo aunque también sea uno de los recursos utilizados en otros procedimientos plásticos. El Dibujo es conocimiento y expresión, pero también comunicación. Recordemos aquí que el concepto de desarrollo profesional intenta superar al concepto de formación continua e incorpora la idea de que "la práctica no es un proceso en el cual sólo se recibe desde fuera una capacitación, sino que supone la implicación directa del que se forma" (Sanjurjo, 2012, p. 38). Si bien es necesario un desarrollo específico de los lenguajes, la enseñanza y el aprendizaje del Dibujo debería incluir una mirada más amplia, referida no sólo a la interdisciplinariedad o pluralidad de medios y lenguajes, sino a la concepción misma del Dibujo, la cual debería avanzar más alla de la expresión y comunicación y potenciarse como un espacio en el cual no sólo se expresa y comunica sino además, posible de ser pensado como un espacio en el que se produce y genera opinión. Esta mirada encierra múltiples posibilidades para que docentes, alumnas y alumnos desarrollen y construyan saberes a través de una metodología de trabajo centrada en la representación gráfica, en la multiplicidad de recursos y medios de producción así como de lenguajes, pero también centrada en las problemáticas contemporáneas que atraviesan al sujeto como persona, teniendo en cuenta todas las estructuras en forma de red con las que interactúa y que -a su vez- lo modifican, lo definen y lo construyen desde lo social, cultural, político, económico, dejando huella en cada una de sus acciones cotidianas, y por ende, en sus producciones artísticas.

La investigación sobre las redes y su alcance intercultural e interdisciplinar, la exploración de modelos colaborativos y participativos, así como la formulación y circulación de saberes compartidos, constituyen un fascinante desafío que abarca no sólo los ámbitos de la comunicación, el arte y la cultura, sino la entera composición y estructura del conocimiento (Bashiron Mendolicchio, 2014, p.4). 
Revista de la Escuela de Ciencias de la Educación, año 11, número 10, enero a diciembre de 2015. Páginas 43-62. ISSN 1851-6297. ISSN EN LINEA 2362-3349. CO-PRESENCIAS Y VINCULACIONES EN RED: LAS RELACIONES INTERDEPENDIENTES EN LA ENSEÑANZA Y EL APRENDIZAJE DEL DIBUJO EN EL NIVEL SUPERIOR UNIVERSITARIO. SUSANA H. MATTANó

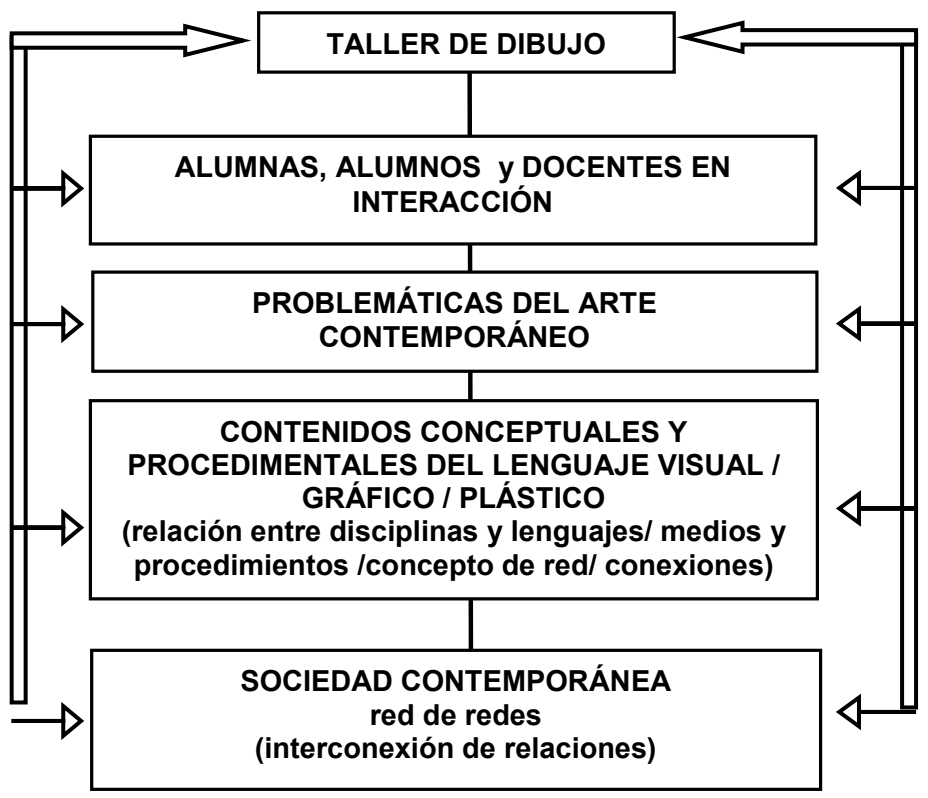

Fig. 1. Taller de Dibujo. Estructura de interrelaciones.

Este abordaje del Dibujo, específicamente en el trayecto final de la Carrera -última etapa de cursado en la cual los supuestos de la formación atraviesan por una definición y profundización del propio desarrollo en la producción plástica- debería contemplar -sin duda alguna-contenidos que aborden las relaciones intertextuales, las metáforas visuales, la biopolítica (4) y las relaciones del arte con el cuerpo y con los campos de la ciencia, la tecnología, la ecología; las problemáticas sociales y urbanas, considerando a las sociedades contemporáneas como interconexión de relaciones y a los sujetos como nodos activos de esas redes en permanente y dinámico intercambio de producciones, lecturas, interpretaciones y resignificaciones.

Los planes de estudio de las carreras artísticas, y en el caso que aquí se aborda, la enseñanza y el aprendizaje del Dibujo en el Nivel Superior Universitario, necesitarían ampliar la mirada y modelar una nueva concepción del sujeto productor de arte por cientos de años centrada en la individualidad, en propio ser y en la propia producción. Es necesario un viraje hacia una mirada que contemple el escenario artístico actual como fluctuante entre distintos paradigmas; la necesaria interrelacción con otros lenguajes y contextos; y además, que considere al Dibujo como eje, medio y fin de la producción artística. En este contexto alumnas y alumnos pueden reflexionar y expresarse comunicando 
Revista de la Escuela de Ciencias de la Educación, año 11, número 10, enero a diciembre de 2015. PÁginas 43-62. ISSN 1851-6297. ISSN EN LINEA 2362-3349. Co-PRESENCIAS Y VINCULACIONES EN RED: LAS RELACIONES INTERDEPENDIENTES

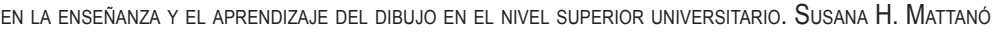

sus propias ideas y experiencias en proyectos artísticos que incluyan un marco teórico que sustente y permita la toma de posición frente a los temas de interés propio y/o general y así ofrecer propuestas que generen y den opinión a través del y en el campo artístico. Las nuevas formas de arte contemplan procesos y proyectos que sitúan "la presencia constante del artista en su proyecto, en el marco que se expone así mismo como persona" (Laddaga, 2006, p.138) con fines que van más allá de un objetivo particular y se despliegan hacia la intensificación y desarrollo de la cooperación social.

Son estos proyectos "seres encabalgados, que forman rizomas o redes" (...) En estos proyectos cada uno de los integrantes se presenta como aquellos que se embarcan en un proceso de articulación en imágenes y discursos de informaciones y problemas inmediatamente vinculados a su circunstancia singular. Claro que el objeto de estos procesos es liberar esa circunstancia de la estricta privacidad. Los participantes de estos proyectos articulan en público aquellos aspectos de su circunstancia que al mismo tiempo dependen de las redes de relaciones en las que se encuentran y que son susceptibles de reelaboración (Ladagga, 2006, pp. 153-155).

Las estrategias didáctico-pedagógicas consideran que si bien, la formación universitaria forma para el mundo del trabajo, no lo hace "únicamente para el trabajo con fines económicos o para la producción de conocimientos, sino también para la construcción de concepciones del mundo, la comprensión de formas de convivencia alternativas, el desarrollo personal, individual y colectivo y la participación política" (Litwin, 2008, p. 26).

Es necesario además, concebir a la producción artística y dentro de ella, a las producciones artísticas realizadas a través del Dibujo como medios que promuevan la crítica, la reflexión, la expresión y comunicación de opiniones y, fundamentalmente, que generen experiencias y espacios que tiendan a la cooperación social, a la búsqueda de soluciones a problemas y al bienestar general.

Bajo la premisa de que una Disciplina -en este caso Dibujo- no se define por el objeto empírico del que se ocupa (como por ejemplo el lenguaje visual, la alfabetidad visual, la representación gráfica; los elementos básicos de la comunicación visual; los principios compositivos; los soportes, materiales y herramientas) sino por el enfoque y la metodología de trabajo con que son abordados los contenidos, los mismos -en los Talleres de Dibujo (y por extensión, la que podría ser aplicada en los Talleres de Pintura, Escultura y Grabado)deberían fluir en forma flexible entre tres ejes de enseñanza y de aprendizaje: el eje Sujeto/s, el eje Texto/s y el eje Contexto/s. 
Revista de la Escuela de Ciencias de la Educación, año 11, número 10, enero a diciembre de 2015. Páginas 43-62. ISSN 1851-6297. ISSN EN LINEA 2362-3349. CO-PRESENCIAS Y VINCULACIONES EN RED: LAS RELACIONES INTERDEPENDIENTES EN LA ENSEÑANZA Y EL APRENDIZAJE DEL DIBUJO EN EL NIVEL SUPERIOR UNIVERSITARIO. SUSANA H. MATTANÓ

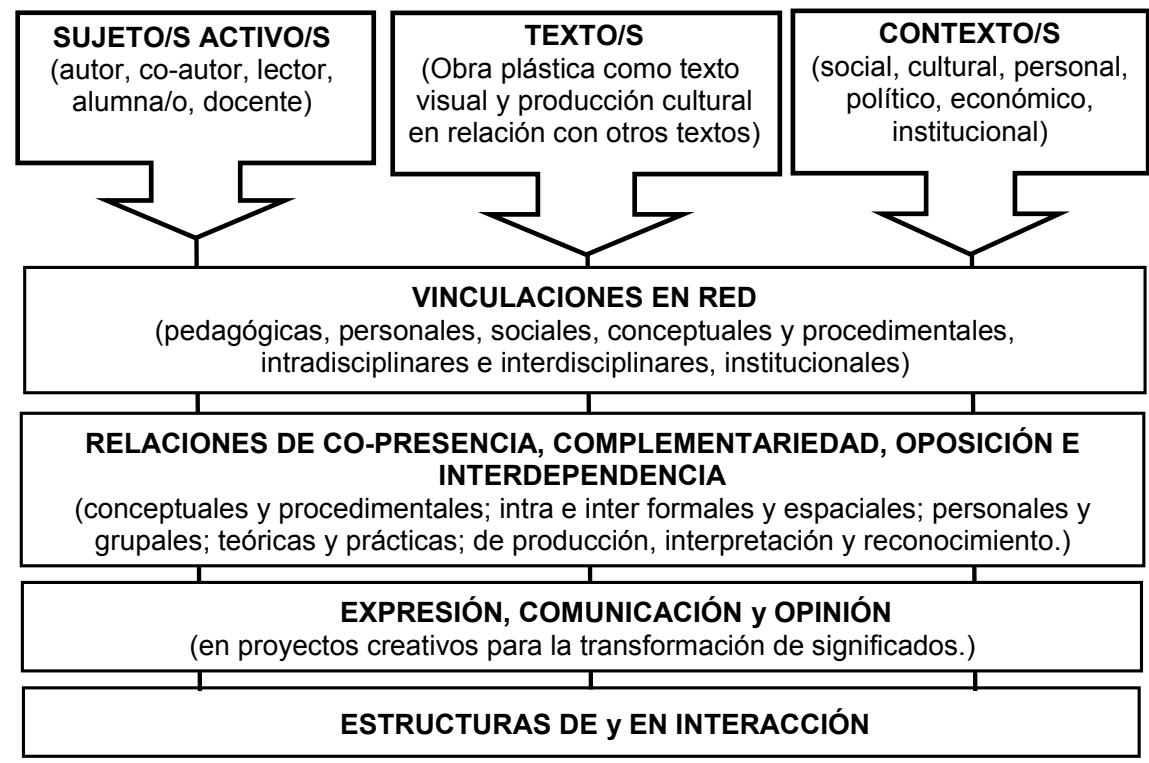

Fig. 2. Vinculaciones de tres ejes de enseñanza y de aprendizaje:

Sujeto/s, Texto/s y Contexto/s.

Estos ejes nombrados en plural, consideran desde su esencia las relaciones múltiples e interdependientes (de co-presencia, opuestas y complementarias) que se dan entre más de un sujeto (entre el o los productores y lectores de la obra; entre autor y co-autores; entre docente y alumnas y alumnos, entre el cuerpo docente y no docente; entre alumnas y alumnos entre sí) como también, a las relaciones interdependientes que se dan entre más de un texto (vinculaciones del texto visual con otros textos y lenguajes) [5] y entre más de un contexto (político, social, económico, personal, institucional, cotidiano, urbano) en una compleja red de intercambios, interrelación, producción, lectura y reconocimiento (6). Es oportuno agregar que estos ejes comprenden parte de la experiencia metodológica de enseñanza y aprendizaje de contenidos desarrollada en el Taller de Dibujo, Comisión "C" de la Carrera de Bellas Artes de la Facultad de Humanidades y Artes de la Universidad Nacional de Rosario y que en una verdadera coordinación horizontal, en la última etapa de la formación de los estudiantes, podrían extenderse a otros espacios curriculares de la carrera, para así lograr tanto en la teoría como en la práctica una verdadera articulación y formación en red (Mattanó, 2014). Podríamos citar aquí, como ejemplos de las vinculaciones en red dentro del campo de la enseñanza y del aprendizaje, tanto la práctica rizomática como 
Revista de la Escuela de Ciencias de la Educación, año 11, número 10, enero a diciembre de 2015. PÁginas 43-62. ISSN 1851-6297. ISSN EN LINEA 2362-3349. CO-PRESENCIAS Y VINCULACIONES EN RED: LAS RELACIONES INTERDEPENDIENTES

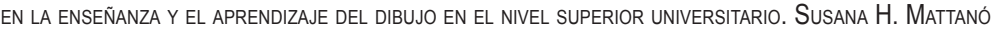

concepción del proceso de enseñanza y de aprendizaje (según la idea de Deleuze y Guattani) como así también el concepto de configuración didáctica de Litwin, quien la define como la

(...) manera particular que despliega el docente para favorecer los procesos de construcción del conocimiento. Esto implica una construcción elaborada en la que se pueden reconocer los modos como el docente aborda múltiples temas de su campo disciplinar que se expresa en el tratamiento de los contenidos, su particular recorte, los supuestos que maneja respecto del aprendizaje, la utilización de prácticas metacognitivas, los vínculos que establece en la clase con las prácticas profesionales involucradas en el campo de la disciplina de que se trata, el estilo de negociación de significados que genera, las relaciones entre la práctica y la teoría que incluyen lo metódico y la particular relación entre el saber y el ignorar (Litwin, 2012, p.97).

Las configuraciones didácticas implican además, los supuestos del proceso de enseñanza-aprendizaje y el contrato pedagógico y las relaciones interdisciplinarias en modos particulares de abordaje del campo disciplinar. Estas posturas son retomadas en el concepto de currículum reticular de Efland en el cual, la práctica niega al docente como centro y a los alumnos como secundarios y se plantea como una situación dinámica de intercambio y conocimiento en continuo crecimiento. Todo ello apunta al proceso de enseñanza y de aprendizaje como proceso de construcción cooperativa, de co-presencia y colaboración entre unos y otros, nutrido por dinámicas de comunicación en el aula; por el pensamiento reflexivo y crítico de los actores implicados; la planificación y redefinición de actividades según los intereses del grupo y el contexto; el cuestionamiento y el autocuestionamiento; la autoevaluación y la evaluación conjunta; las prácticas educativas democráticas basadas en la inclusión del otro y el intercambio con el otro.

Hay fuerzas entre nosotros que nos acercan o nos alejan de los demás, que nos hacen preferir un grupo a otro. En ocasiones nuestra personalidad desaparece y asemejamos más un grupo de insectos sociales que una sociedad de individuos propiamente «individuales». Quizá no somos más que partículas transportadoras de interacciones, fotones que entrelazan su ser a otros y participan de una red de relaciones que veríamos extenderse a toda la humanidad si no tuviéramos tan corta vista (Brinones, C.; Manrubia, S.; MartínGago, J. A., 2009).

Concebir e interpretar la educación en forma de rizoma, mapa, configuración o construcción cooperativa es traducirla al concepto contemporáneo de vinculación en red y de estructura de interacción, caraterizada por la for- 
Revista de la Escuela de Ciencias de la Educación, año 11, número 10, enero a diciembre de 2015. Páginas 43-62. ISSN 1851-6297. ISSN EN LINEA 2362-3349. Co-PRESENCIAS Y VINCULACIONES EN RED: LAS RELACIONES INTERDEPENDIENTES

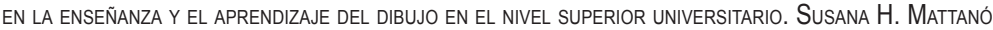

mación de nuevas estructuras o nuevas redes que se nutren por los múltiples aportes y producciones heterogéneos en conexión entre docentes, alumnas y alumnos. Esta concepción de enseñar y aprender en red implica también una correlación entre contenidos, procesos y producciones teóricas y prácticas que aborden la solución de situaciones problemáticas ya que resolver un problema implica pensar en sus consecuencias, considerar soluciones de problemas similares; elaborar hipótesis; desarrollar argumentos; construir explicaciones; trabajar con andamiajes y de forma independiente; descubrir nuevos significados y además, plantearse nuevos problemas para intentar resolverlos. Por ello, el Dibujo en el Nivel Superior Universitario debe ser concebido como un espacio teórico y práctico, dejando de lado la unívoca concepción práctica que sólo involucra el hacer del dibujo como actividad manual o de representación visual. El Dibujo es un espacio que fusiona teoría y práctica, no sólo desde los conceptos teóricos y prácticos del lenguaje visual, sino desde los planteos teóricos y prácticos que involucran a los Sujeto/s, a los Texto/s y a los Contexto/s.

A continuación se presenta como ejemplo, parte de la experiencia de enseñanza y aprendizaje desarrollada en el Taller de Dibujo V, Comisión C, de la Escuela de Bellas Artes, Facultad de Humanidades y Artes, Universidad Nacional de Rosario. Se trata de un proyecto de trabajo que reconoce, valoriza e integra textos y lenguajes; instala nuevos significados y que expresa, comunica y genera opinión a través de una producción artística como síntesis de la red de redes que nos contectan, nos atraviesan y nos construyen constantemente como sujetos creativos en interdependencia e interrelación.

La planificación de los prácticos del Taller de Dibujo V, Comisión C, son pensados como proyectos creativos que promuevan el debate sobre problemáticas del arte contemporáneo; incluyan las miradas del conjunto sobre los ejes sugeridos de trabajo e integren contenidos conceptuales, procedimentales, actitudinales. Contemplan libertad en las propuestas creativas con diversidad de procesos de configuración, selección de soportes y materiales convencionales y no convencionales del Dibujo. Proponen además, la comunicación de ideas e intereses en proyectos que sean desarrollados y/o concretados en cualquier otro Taller que curse el alumno (Pintura, Grabado, Escultura) con el fin de promover la construcción de saberes integrados e interdisciplinarios y reforzar las conexiones en red. 
Revista de la Escuela de Ciencias de la Educación, año 11, número 10, enero a diciembre de 2015. PÁginas 43-62. ISSN 1851-6297. ISSN EN LINEA 2362-3349. CO-PRESENCIAS Y VINCULACIONES EN RED: LAS RELACIONES INTERDEPENDIENTES

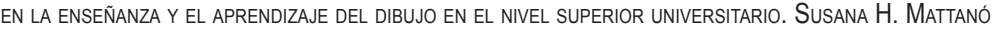

\section{Contenidos conceptuales del Proyecto presentado como ejemplo:}

Intertextualidad. Tipología. Relaciones presentes en las obras plásticas.

Encuadre teórico:

Exploración del concepto de intertexto como aquél que orienta y condiciona la interpretación de todo el texto visual; como desafío hermenéutico y como relación con el pasado, la memoria, la cultura y la transformación del presente a través de la interactividad textual permitiendo nuevas visiones del mundo. El concepto de intertextualidad implica el de huella y no se concibe como intento de copia, sino como presencia de otros textos en un texto nuevo, el cual los recibe, los absorbe y los integra originando una nueva unidad de significado que es reflejo del concepto de redes y de relaciones interdependientes.

\section{Eje de trabajo:}

Texto/s.

Como parte de relaciones interdependientes, el eje Texto/s implica el trabajo simultáneo con los otros dos (Sujeto/s y Contexto/s) ya que los textos son producidos y leídos por sujetos activos, en contextos y marcos culturales particulares y diferentes.

\section{Objetivos:}

Crear una nueva unidad de significado en un proyecto creativo de expresión, comunicación y que genere opinión.

Seleccionar un contenido temático que aborde la problemática del cuerpo, la de género y la de relaciones interdependientes.

\section{Alumna: Marisol González}

Año: 2011

Espacio curricular: Taller de Dibujo V. Comisión C. Departamento de Expresión Visual. Escuela de Bellas Artes. Facultad de Humanidades y Artes. Universidad de Rosario.

Al trabajar los intertextos en los textos visuales, la alumna Marisol González, aborda la red intertextual que nos atraviesa, citando, refiriendo y aludiendo a las obras "Identical Twins" (Fig. 3) e "Identical Nicolas" (Fig. 4) de la fotógrafa norteamericana Diane Arbus (1923-1971) y de la artista plástica rosarina Nicola Constantino (1964) respectivamente. 
Revista de la Escuela de Ciencias de la Educación, año 11, número 10, enero a diciembre de 2015. PÁginas 43-62. ISSN 1851-6297. ISSN EN LINEA 2362-3349. CO-PRESENCIAS Y VINCULACIONES EN RED: LAS RELACIONES INTERDEPENDIENTES EN LA ENSEÑANZA Y EL APRENDIZAJE DEL DIBUJO EN EL NIVEL SUPERIOR UNIVERSITARIO. SUSANA H. MATTANÓ

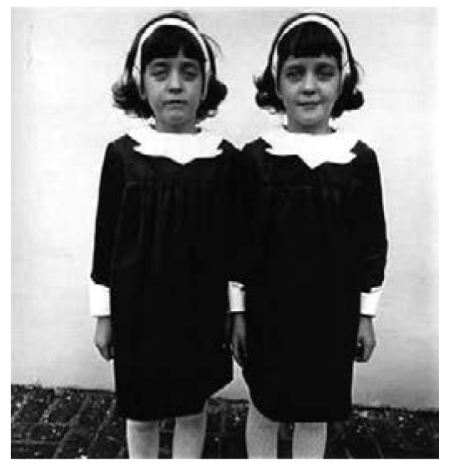

Fig. 3. "Identical Twins." Diane Arbus (1923 - 1971)

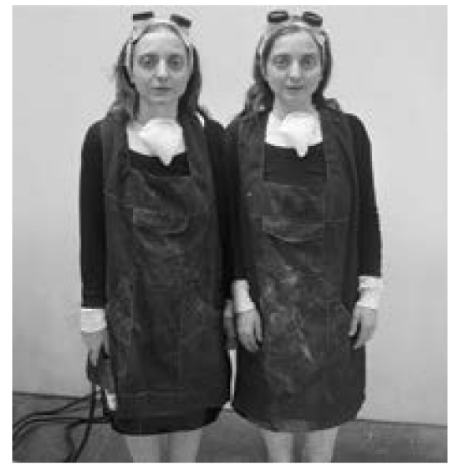

Fig. 4. "Identical Nicolas." Nicola Constantino (1964)

Marisol expande la red de intertextos que nos atraviesa al plantear como punto de partida de su producción plástica la obra "Nicola cita Arbus" (Fig. 5) haciendo referencia a las autoras desde el título. Cita en superposición las fotografías de Arbus y de Constantino a la manera de un fotomontaje de fotogramas y hace una referencia clara a ambas en el título de su propia obra, ofreciendo así un juego y un interrogante sobre la problemática de género, la autoría y la fusión de ideas, saberes e intercambios en las expresiones contemporáneas.

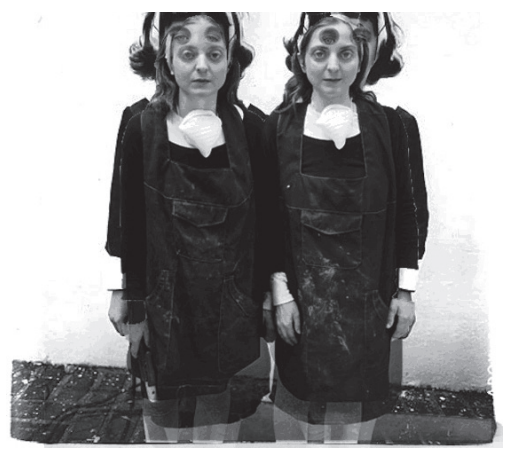

Fig. 5. "Nicola cita Arbus". Marisol González.

Taller de Dibujo V, Comisión C, año 2011

Desarrolla una serie de Dibujos titulados Intertextos (Fig. 6) en la cual, el cruce intertextual está dado por alusiones y citas a Arbus y Constantino, a las gemelas como doble a través de la actitud corporal y las expresiones de los rostros: uno con y otro sin sonrisa; a la vestimenta y a la representación gráfica de la misma toma fotográfica. 

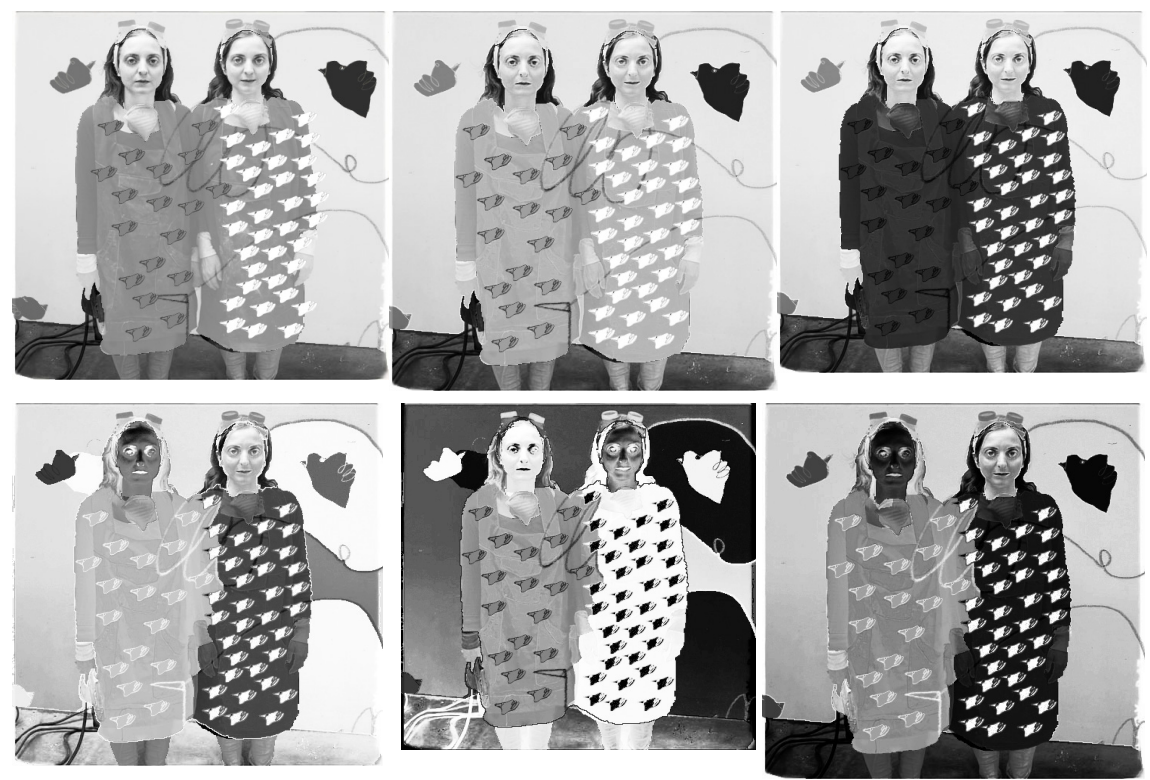

Fig. 6. Serie "Intertextos". Marisol González.

Taller de Dibujo V, Comisión C, año 2011

Avanza aún más al aludir -a través del dibujo- a la fotografía de una mujer con joroba de Arbus (Fig. 7) que instala junto a la cita parcial de "Identical Nicolas" (Fig. 8). El tratamiento gráfico provoca un borramiento del doble; las figuras antes gemelas, con poses y actitudes idénticas, ya no son "Identical Nicolas" de Constantino, ni tampoco "Identical Twins" de Arbus. Dibujo, fotografía y digitalización se funden en una nueva obra con un nuevo significado, ofreciendo un juego de líneas; formas y espacios positivos y negativos, en donde Marisol cita a la obra de Constantino y alude a la de Arbus pero ahora, se trata de su propia obra. Es inevitable aquí dejar de reconocer las primeras consideraciones de este escrito, sobre el borramiento de límites y fronteras que opera en nuestra vida globalizada y en permanente conexión en red. 
Revista de la Escuela de Ciencias de la Educación, año 11, número 10, enero a diciembre de 2015. PÁginas 43-62. ISSN 1851-6297. ISSN EN LINEA 2362-3349. CO-PRESENCIAS Y VINCULACIONES EN RED: LAS RELACIONES INTERDEPENDIENTES EN LA ENSEÑANZA Y EL APRENDIZAJE dEL DIBUJO En EL NIVEL SUPERIOR UNIVERSITARIO. SusANA H. MATtANó

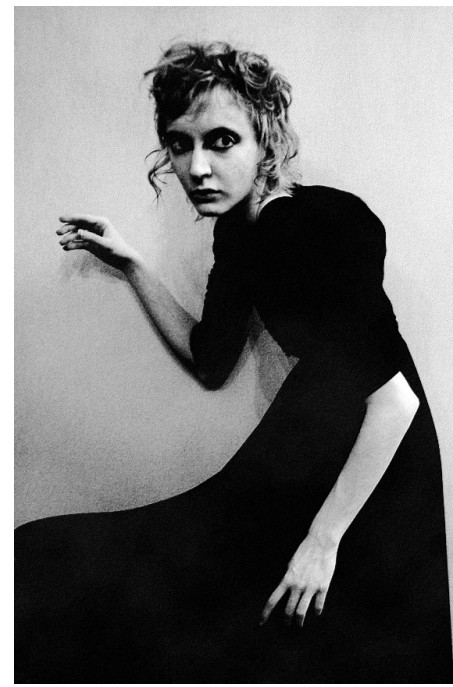

Fig. 7. Fotografía de Diane Arbus

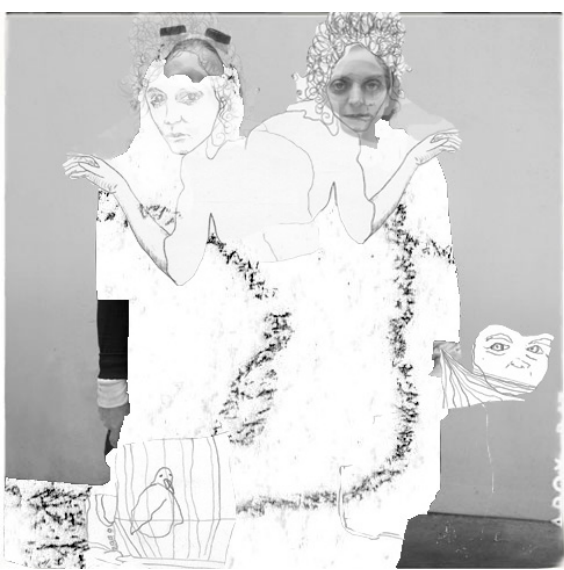

Fig. 8. "Identical Nicolas". Marisol González. Taller de Dibujo V, Comisión C, año 2011

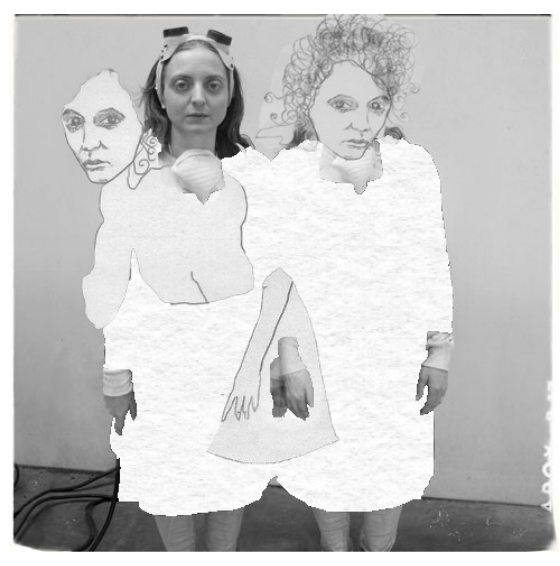

Fig. 9. "Identical Nicolas". Marisol González. Taller de Dibujo V, Comisión C, año 2011 
Revista de la Escuela de Ciencias de la Educación, año 11, número 10, enero a diciembre de 2015. PÁginas 43-62. ISSN 1851-6297. ISSN EN LINEA 2362-3349. Co-PRESENCIAS Y VINCULACIONES EN RED: LAS RELACIONES INTERDEPENDIENTES

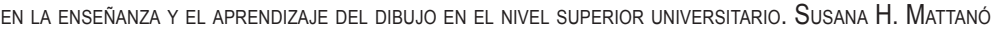

\section{Conclusiones}

Si bien se observa permeabilidad en cuanto a la presencia de distintos lenguajes y disciplinas en las prácticas de producción artística en la enseñanza y el aprendizaje de contenidos en la Carrera de Bellas Artes de la Facultad de Humanidades y Artes de la Universidad Nacional de Rosario, los planes de estudio diferencian los espacios curriculares según una concepción moderna de las artes y en una estructura de Talleres: Taller de Dibujo, Taller de Grabado, Taller de Pintura, Taller de Escultura. De este modo, se refleja una mirada esencialmente práctica de cada una de las disciplinas que apunta a la especificidad en cada una ellas. Si bien, cada uno de los Talleres se instala como un espacio curricular para el desarrollo de saberes específicos, para el conocimiento, desarrollo y profundización del lenguaje visual y la práctica de medios y procedimientos adecuados; en la práctica, suelen estimularse las producciones artísticas sólo desde la expresión creativa del estudiante sin proponer realizar una contextualización referida a las problemáticas contemporáneas del arte; la interdisciplinariedad; los principios de interdependencia e interconexión; la co-presencia de distintos lenguajes en las producciones artísticas; la fusión de medios y en definitiva, el reflejo de los paradigmas culturales, sociales y políticos que atraviesan a los sujetos como individuos, como alumnos, como lectores o productores de arte. Parecería ser que -por lo general-en los Talleres se hace arte y sólo se reflexiona sobre los paradigmas del arte contemporáneo en los espacios curriculares de teoría, historia y crítica, cuando la reflexión es esencia misma de la producción artística. Ahora bien, es necesario reconocer que existen relaciones de co-presencia y de interdependencia tanto en los contenidos específicos del lenguaje visual (para citar sólo algunos: relación figura-fondo; espacios y formas positivas y negativas; colores cálidos y fríos; lo presente y lo ausente en la representación y en el significado; lo real y lo ilusorio; etc.); entre los actores; en los procesos y relaciones de enseñanza y aprendizaje; como así también en su necesaria fundamentación y relación teórico-práctica.

Ante este cuadro de situación, sólo las estrategias didácticas y propuestas metodológicas de contenidos en relación al mundo, que atiendan circunstancias que nos atraviesan y, además, contemplen los cambios continuos de la vida cotidiana personal y en sociedad, permitirán una formación en el campo del Dibujo en el Nivel Superior Universitario que apunte a las concreción de producciones artísticas con fines que superen lo contemplativo y el placer estético como únicos objetivos.

El desarrollo de una práctica de enseñanza y aprendizaje del Dibujo en el Nivel Superior Universitario, además de desarrollar los contenidos específicos del lenguaje gráfico-visual debe contemplar la formación de alumnas y alumnos para ser capaces de dar y generar opinión a través de las prácticas artísticas; debe formarlos para ser capaces de fundamentar las propuestas de expresión y comunicación a través del Dibujo -en un marco teórico concreto- y poder 
Revista de la Escuela de Ciencias de la Edducación, año 11, número 10, enero a diciembre de 2015. PÁginas 43-62. ISSN 1851-6297. ISSN EN LINEA 2362-3349. Co-PRESENCIAS Y VINCULACIONES EN RED: LAS RELACIONES INTERDEPENDIENTES EN LA ENSEÑANZA Y EL APRENDIZAJE DEL DIBUJO EN EL NIVEL SUPERIOR UNIVERSITARIO. SUSANA H. MATTANó

referirlas tanto a las problemáticas del arte contemporáneo como a aquellas que los atraviesan como sujetos en interacción y en despligue permanente de relaciones de oposición, complementariedad e interdependencia; como actores de los intercambios sociales, políticos y culturales y como estudiantes capacitándose dentro y para el mundo del cual forman parte. Todo ello en conjunto permitirá redimensionar el valor de los estudios artísticos; el de la enseñanza de las artes en general, y el de la enseñanza y aprendizaje del Dibujo en el Nivel Superior Universitario en particular. Especialmente, se revalorizará el rol de las prácticas del arte en nuestra comunidad y además, se resignificará la función del arte en nuestras vidas y en la de los otros, propiciando nuevas mutaciones culturales y sociales en red basadas en la cooperación social, la solución de problemas y el bien común.

Treinta radios convergen en el buje de una rueda,

y es ese espacio vacío lo que permite al carro cumplir su función.

Modelando el barro se hacen los recipientes, y es su espacio vacío lo que los hace útiles.

Puertas y ventanas se abren en las paredes de una casa, y es el espacio vacío lo que permite que la casa pueda ser habitada.

Lo que existe sive para ser poseído.

Lo que no existe sirve para cumplir una función

(Lao Tse, 1998, p.47).

\section{Notas bibliográficas}

(1) Refiriéndose al lenguaje cinematográfico de Peter Watkins, Ladagga (2006, p.157) señala que este cineasta "supone que en ciertas condiciones la película tiene su eficacia verdadera: donde la colectividad misma que la ha constituido está presente, aunque no sea en delegación, en la sala donde la película se muestra" y aspira a una película "que actúe como mediadora entre nudos de una red o tejido de particularidades que se extiende." Esta mirada del lenguaje del cine grafica la señalada para el lenguje plástico y podría extenderse a la obra de arte entendida desde diversas manifestaciones ya sean respondan a categorías de simbolización modernas o bien a las contemporáneas entendidas más como experiencias parcitipativas.

(2) A partir de los años 70 el pensamiento contemporáneo en las artes ha sido influido por la noción de postmodernidad, la cual marca la incredulidad respecto de los grandes relatos. En su artículo La Post-Postmodernidad: Paradigmas Culturales para el Siglo XXI, Marisa Gómez señala que la posmodernidad "se ve a sí misma como la época de todos los fines (de la historia, del arte, de las certezas, del universalismo, etc.) (...) como teoría y como paradigma cultural, orbita entorno a ideas como la crisis del significado, del sujeto, de la realidad, de los valores, del estado, etc.". La autora comenta además que parecería ser que la lógica cultural postmoderna y el postmodernismo como estética dominante, toma un nuevo rumbo -a partir de finales de la década de los 2000-. El mismo, parecería estar marcado por una sensibilidad generalizada "por la necesidad de averiguar, definir y dar nombre a lo que viene después, a la nueva lógica cultural que 
Revista de la Escuela de Ciencias de la Educación, año 11, número 10, enero a diciembre de 2015. PÁginas 43-62. ISSN 1851-6297. ISSN EN LINEA 2362-3349. Co-PRESENCIAS Y VINCULACIONES EN RED: LAS RELACIONES INTERDEPENDIENTES EN LA ENSEÑANZA Y EL APRENDIZAJE dEL DIBUJO En EL NIVEL SUPERIOR UNIVERSITARIO. SusANA H. MatTANó

se está configurando bajo nuestra -todavía perpleja- mirada a los cambios cultturales y sociales que han acompañado al nuevo siglo.

(3) Con respecto a la disipación del autor en estas nuevas expresiones, Laddaga (2006, pp.138-145) comenta que se trata de algo distinto a la muerte del autor planteada por Foucault, dejando de tener que ver la figura del autor con el exhibicionismo propio de la modernidad, y configurándose como una disipación que se diluye en la interacción y la cooperación social.

(4) La Biopolítica como paradigma contemporáneo de poder acciona sobre necesidades y relaciones de la población y abarca cuestiones territoriales, demográficas, ecológicas, económicas, tecnológicas, de seguridad, educación y salud como formas de gestión y regulación del poder político sobre los cuerpos y la vida de los individuos. Michel Foucault analiza un tipo de poder -el biopoder- el cual opera sobre los cuerpos vivientes, los afectos y las relaciones humanas; sobre el cuerpo social, sus tramas y redes y cuya función ya no es matar, sino invadir la vidas de los individuos. El término biopolítica proviene del campo de la genética; vincula a los avances tanto en ese ámbito como en el de la biología, en el de la legislación sobre el aborto y la eutanasia, refiere al peligro acerca de la conservación del medioambiente y a los esfuerzos de los países por incrementar las medidas de seguridad en temas relativos a la inmigración o a las guerras preventivas. Andrea Giunta (2009, p.251) señala que las vinculaciones entre política y vida biológica "pueden ser negativas, cuando buscan dominar la vida y reproducirla bajo el control de variados sistemas sociales (legales, políticos, administrativos, etc.), o tener una finalidad positiva, cuando su propósito es emancipar la vida de la explotación, el manejo o la dominación."

(5) Es necesario comprender que la obra como texto es un campo de relaciones intertextuales íntimamente relacionada con el marco cultural de cada sujeto, y también pensar que la noción de texto, ampliamente desarrollada por Umberto Eco, desde la óptica de Julia Kristeva, es abordada desde la noción de experiencia. Kristeva manifiesta su interés por "el laboratorio donde se producen los textos", haciendo clara alusión al hecho artístico como experiencia; en el cual la producción de un texto implica el necesario cuestionamiento de "la manera de sentir, la sexualidad, el lenguaje" como "cuestionamiento de lo antiguo y posterior surgimiento de lo nuevo", que necesariamente implica un "conocimiento técnico del texto". Kristeva (2011, pp.6-7). Experiencia y conocimiento técnico que, sin lugar a dudas, es imprescindible tanto en la producción y en la lectura (recepción/reconocimiento) de la obra de arte.

(6) El concepto de revuelta -entendido desde su etimología como pasar hacia atrás y volver hacia el futuro- es aportado por Julia Kristeva, quien alude a "una memoria fuerte de la transformación (...). El sentido profundo de la revuelta tiene que ver con revalorizar los antiguos valores para que surjan otros, nuevos" Kristeva (2011, p.8). Desde esta mirada, también podemos señalar la co-presencia -en este caso de valores-necesaria también en los procesos de transformación y cambio.

(7) Brinones, C.; Manrubia, S.; Martín-Gago, J. A. (2009). Atrapados en la red: nanomundo, vida, sociedad. En banquete_ nodos y redes, Sociedad Estatal para la Acción Cultural Exterior de España SEACEX.

\section{Referencias Bibliográficas}

- Bashiron Mendolicchio, H. (2014). Modelos colaborativos y Saberes compartidos. Redes, Creatiatividad e Interculturalidad. Interartive, a platform for contemporary art and thought. Fecha de recupero 31 de octubre de 2014. 
Revista de la Escuela de Ciencias de la Edducación, año 11, número 10, enero a diciembre de 2015. PÁginas 43-62. ISSN 1851-6297. ISSN EN LINEA 2362-3349. CO-PRESENCIAS Y VINCULACIONES EN RED: LAS RELACIONES INTERDEPENDIENTES EN LA ENSEÑANZA Y EL APRENDIZAJE DEL DIBUJO EN EL NIVEL SUPERIOR UNIVERSITARIO. SUSANA H. MATTANÓ

- Brinones, C.; Manrubia, S.; Martín-Gago, J. Á. (2009). "Atrapados en la red: nanomundo, vida, sociedad". En banquete_nodos y redes, Sociedad Estatal para la Acción Cultural Exterior de España: SEACEX.

- $\quad$ Giunta, Andrea (2009). Glosario. Poscrisis. ARTE ARGENTINO DESPUÉS DE 2001. Buenos Aires: Siglo veintiuno editores.

- Gómez, M. (2014). La Post-Postmodernidad: Paradigmas Culturales para el Siglo XXI. Interartive, a platform for contemporary art and thought. Fecha de recupero $27 \mathrm{de}$ octubre de 2014.

- $\quad$ Kristeva, J. (2011). El lenguaje de la revuelta. Revista Ñ, Revista de Cultura. Entrevista. Clarín, 24/11.

- Laddaga, R. (2010). Estética de laboratorio. Buenos Aires: Adriana Hidalgo.

- (2006). Estética de la emergencia. La formación de otra cultura de las artes. Buenos Aires: Adriana Hidalgo.

- $\quad$ Lao Tse. (1998). Tao Te Ching. Barcelona: Integral. (2a edición)

- Litwin, E. (2012). Las configuraciones didácticas. Una nueva agenda para la enseñanza superior. Buenos Aires: Paidós. (6a reimpresión)

- Ohlenschläger, K. (2009). "Nodos y redes". En Banquete_nodos y redes, Sociedad Estatal para la Acción Cultural Exterior de España: SEACEX.

- Sanjurjo, L. (2012). "Razones que fundamentan nuestra mirada acerca de la formación en las prácticas". En Sanjurjo, L. (Coord.). Los dispositivos para la formación en las prácticas profesionales. Rosario: Homo Sapiens (1ª reimpresión).

- Zuñiga, R. (2008). La demarcación de los cuerpos. Tres textos sobre arte y biopolítica. Santiago de Chile: Ediciones/metales pesados. 